\title{
LA-UR-18-29092
}

Approved for public release; distribution is unlimited.

Title: $\quad$ Using Aquatic Insects for Water Quality Biomonitoring

Author(s): $\quad \begin{array}{ll}\text { Stanek, Jenna } \\ \text { Gaukler, Shannon Marie } \\ & \text { Berryhill, Jesse Tobias }\end{array}$

Intended for: Educational Outreach

Issued: 
Disclaimer:

Los Alamos National Laboratory, an affirmative action/equal opportunity employer, is operated by the Los Alamos National Security, LLC for the National Nuclear Security Administration of the U.S. Department of Energy under contract DE-AC52-06NA25396. By approving this article, the publisher recognizes that the U.S. Government retains nonexclusive, royalty-free license to publish or reproduce the published form of this contribution, or to allow others to do so, for U.S. Government purposes. Los Alamos National Laboratory requests that the publisher identify this article as work performed under the auspices of the U.S. Department of Energy. Los Alamos National Laboratory strongly supports academic freedom and a researcher's right to publish; as an institution, however, the Laboratory does not endorse the viewpoint of a publication or guarantee its technical correctness. 


\section{Using Aquatic Insects for Water Quality Biomonitoring}

\section{Jenna Stanek, Shannon Gaukler, and}

Jesse Berryhill

\section{Sept 25,2018}




\section{Aquatic Insect Life Cycle}

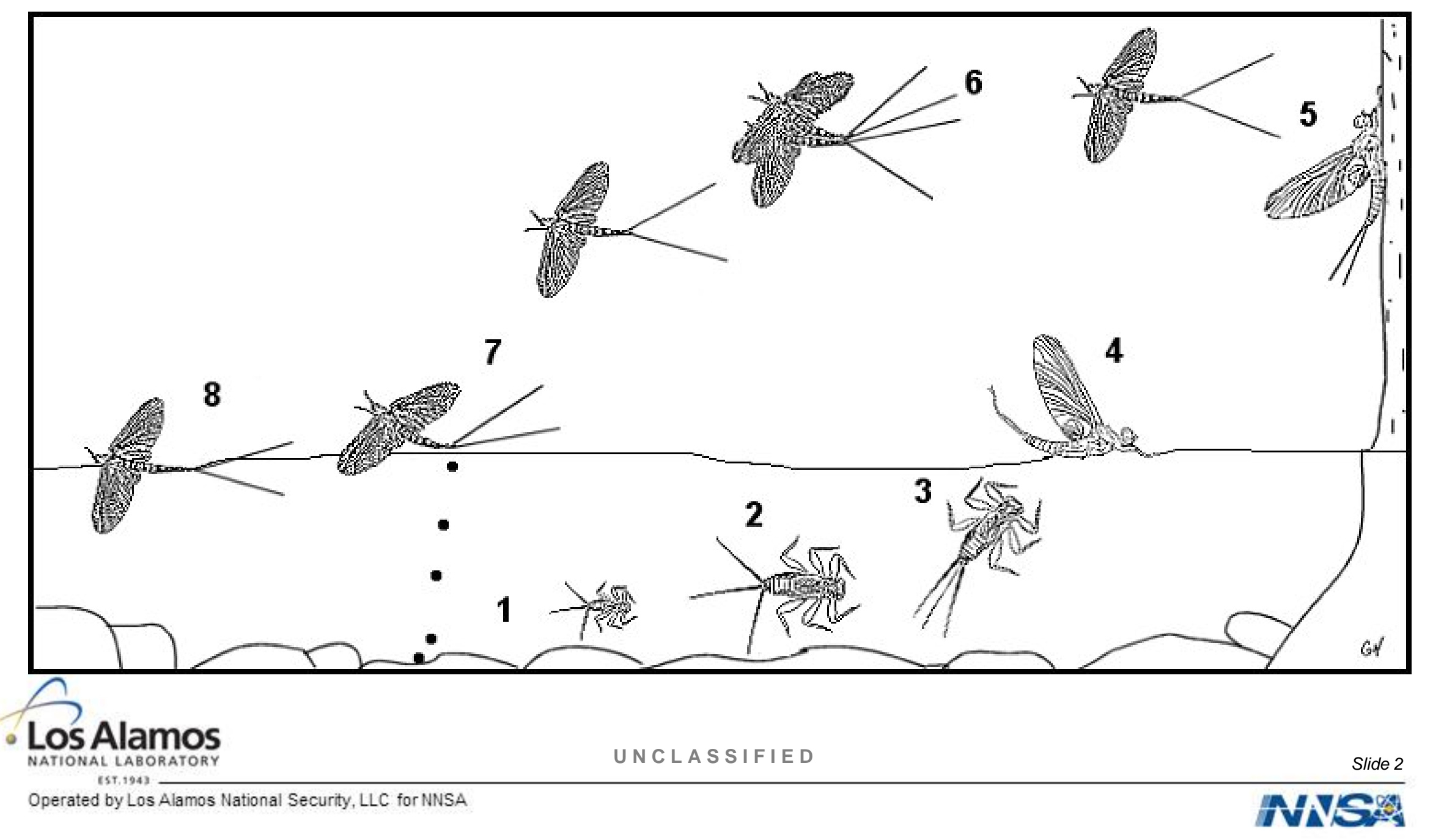




\section{How do aquatic insects breathe?}

- Gills

- Breathing Tubes

- Air bubbles
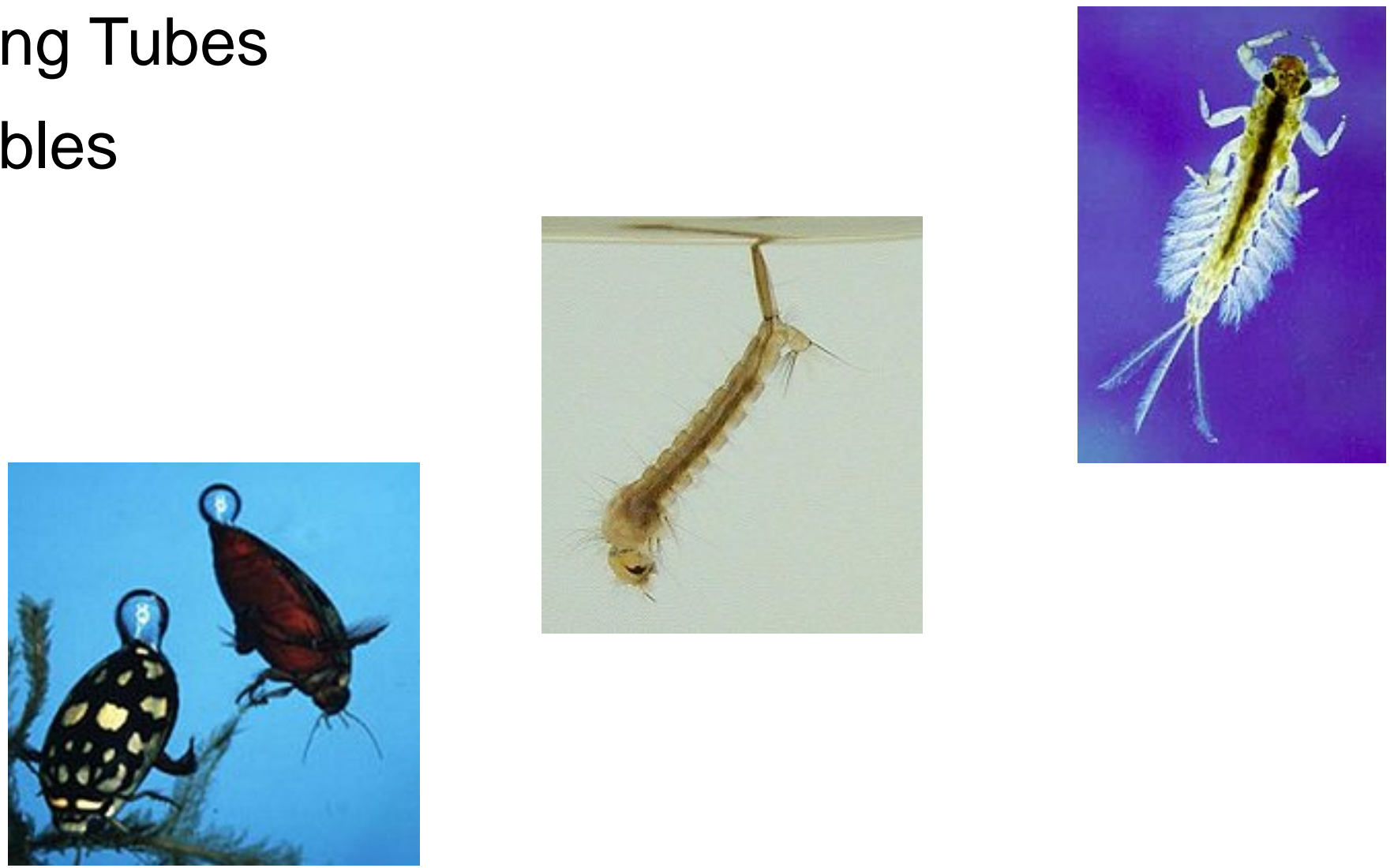


\section{What do aquatic insects eat?}

- Predators - Eat other insects

- Shredders - Eat algae/leaves

- Grazers/Scrapers - Eat algae

- Filterers - Fine particles in water

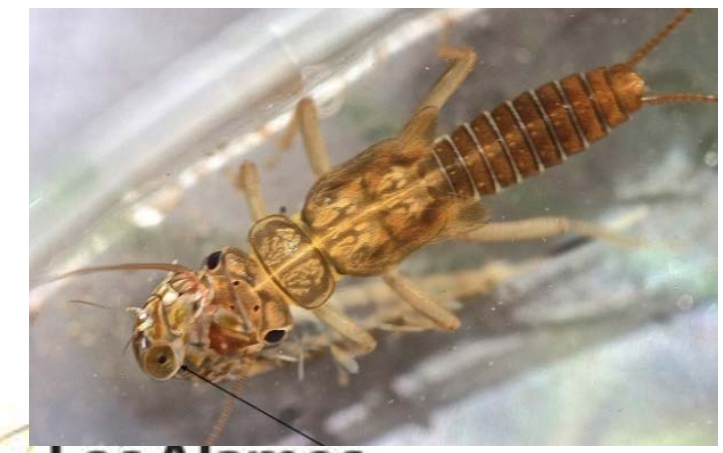

- Los Alamos

NATIONAL LABORATORY

Operated by Los Alamos National Security, LLC for NNSA

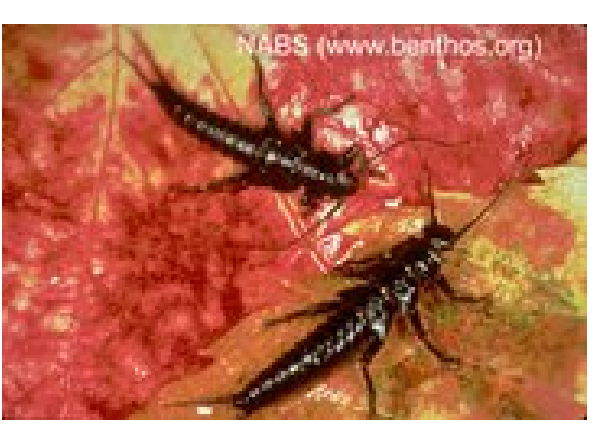

UNCLASSIFIED

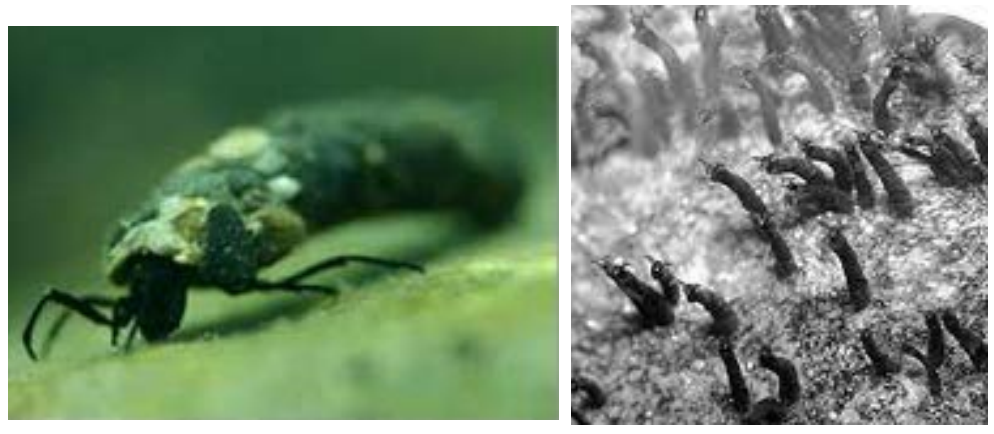

Slide 4

NVS 


\section{Why is water quality important?}

- Drinking water

- Wildlife

- Recreation

- Agriculture
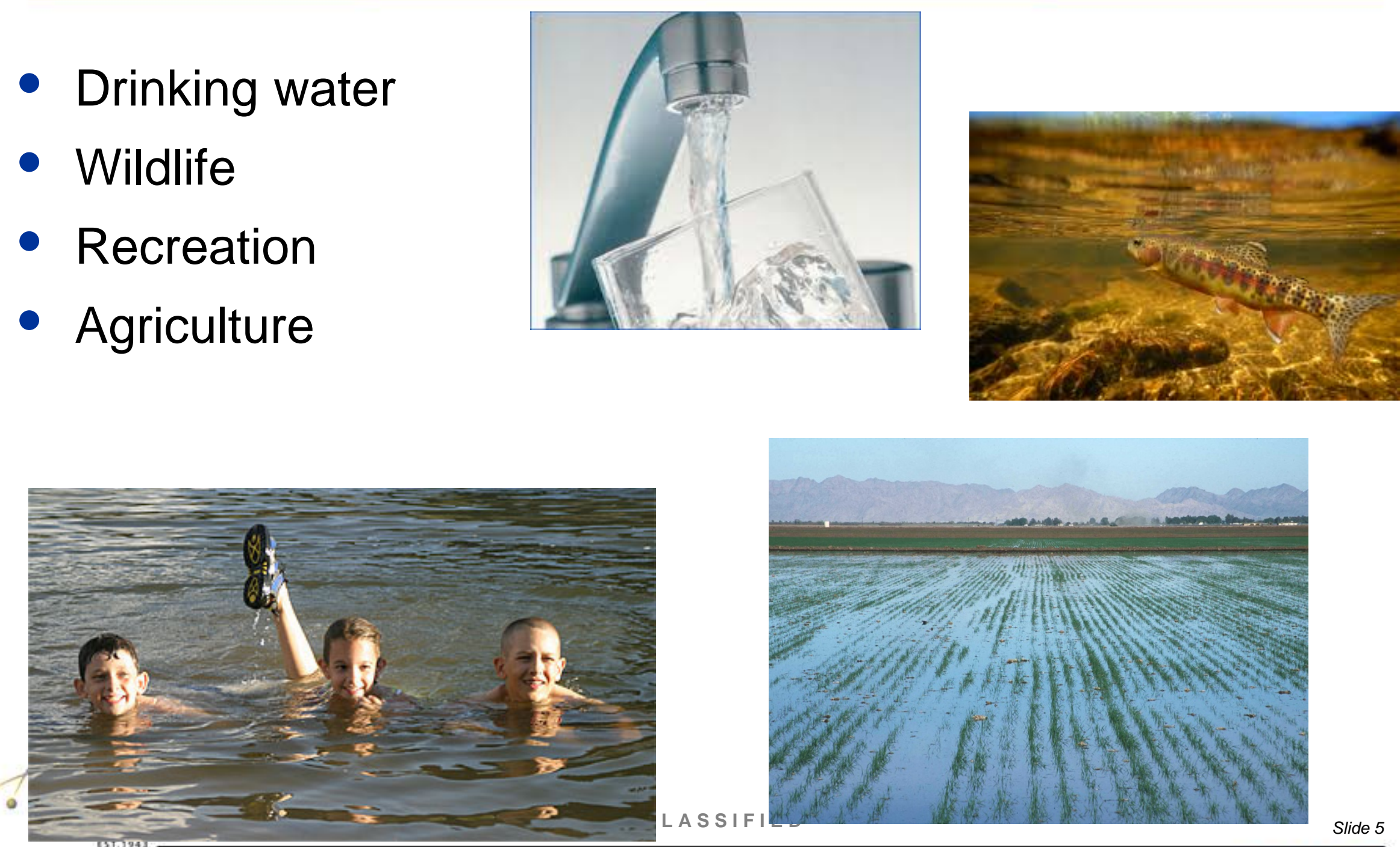


\section{What affects water quality?}

- Urbanization

- Logging

- Pesticide/fertilizer runoff

- Mining

- Non-point source pollution

- Erosion

- Waste water discharges 


\section{Why aquatic insects?}

- Ubiquitous (found everywhere)

- Diverse, with varying tolerances

- 'Easy' to sample and identify

- Short life cycles - quick responses

- Spend majority of their lives in water

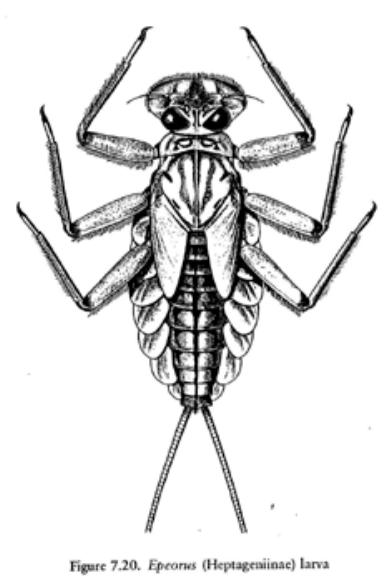

Los Alamos

NATIONAL LABORATORY

$$
\text { (ST, 1943) }
$$

Operated by Los Alamos National Security, LLC for NNSA
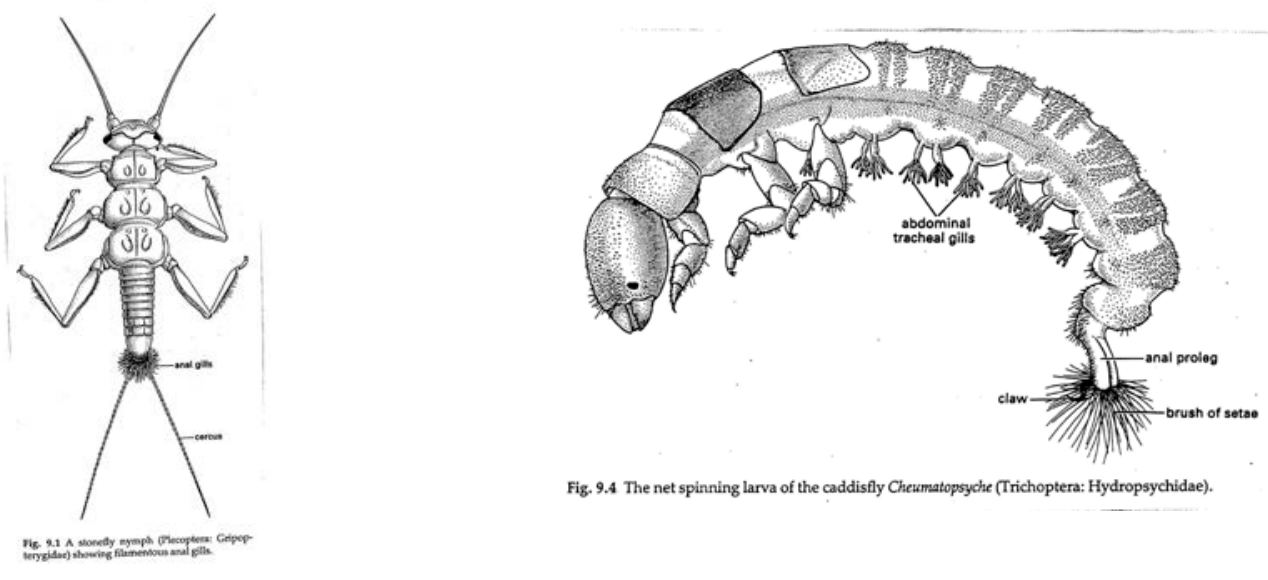


\section{Tolerance Values}

- Indicator organisms - will show if there is a change in habitat quality

- Range of tolerance - Certain insects are pollution tolerant others are very sensitive
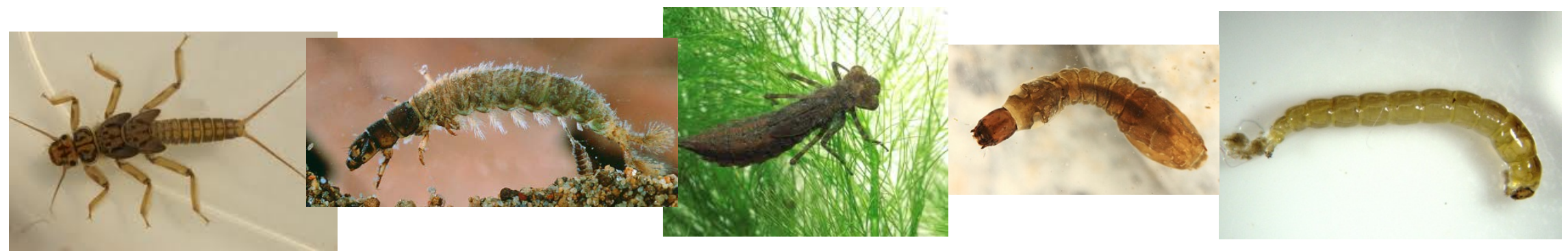

Pollution sensitive
Pollution tolerant 


\section{Assessing water quality}

- Collect a sample of aquatic insects

- Identify aquatic insects

- Add up assigned tolerance values for the sample

- Water quality rating given based on tolerance values

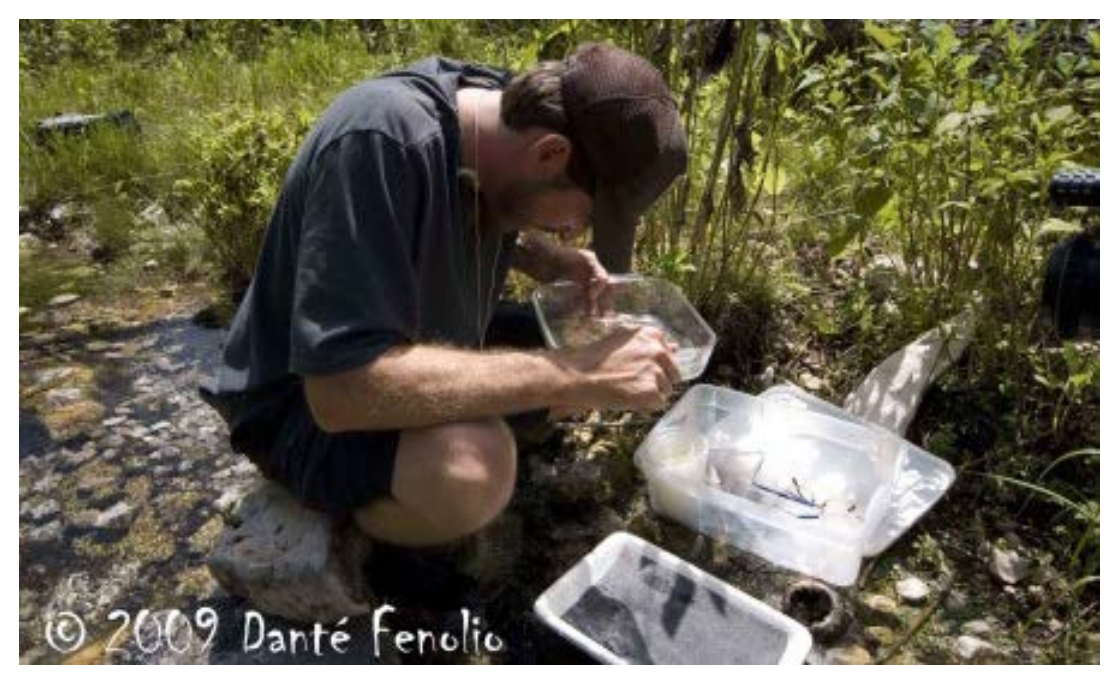




\section{Pollution Sensitive Insects}

- Stoneflies, Mayflies, Caddisflies, and others...
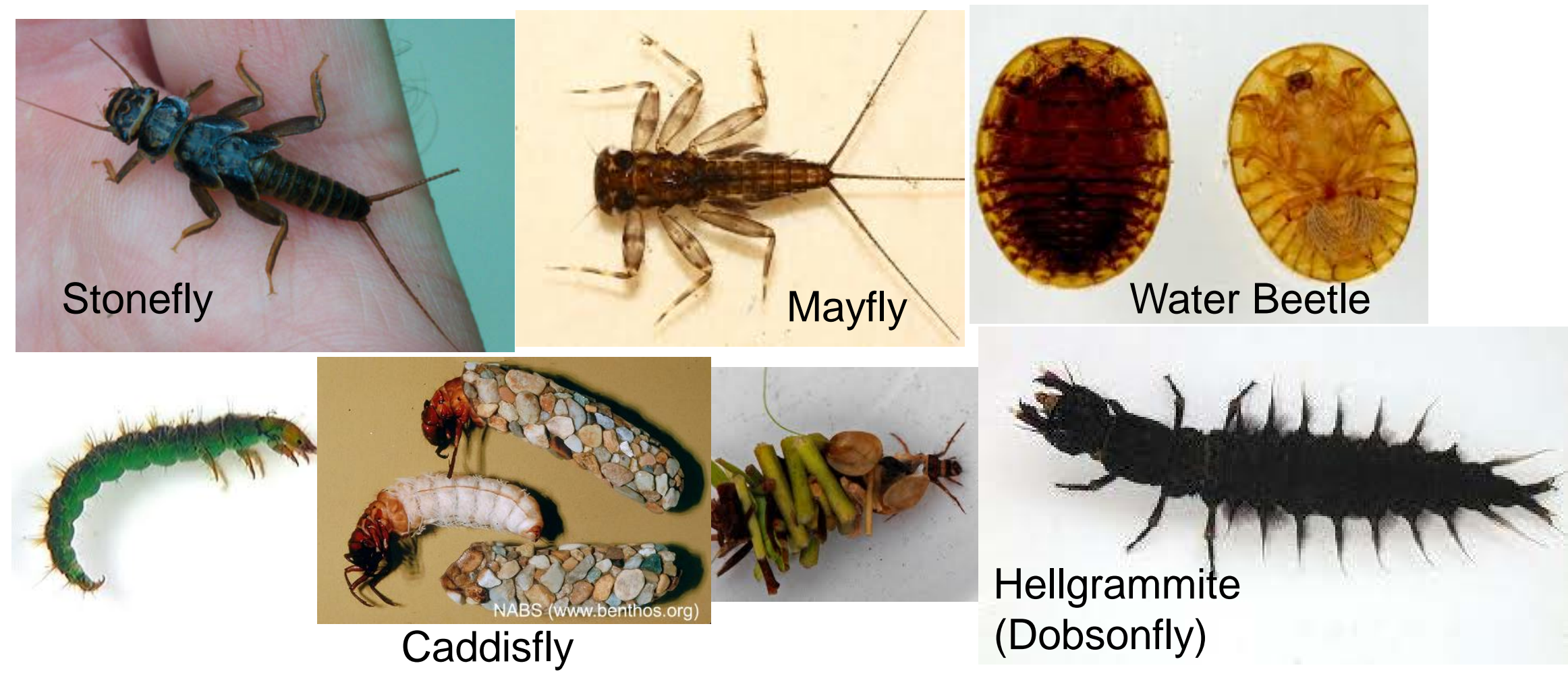


\section{In between tolerance}

- Craneflies, dragonflies, damselflies, and more...
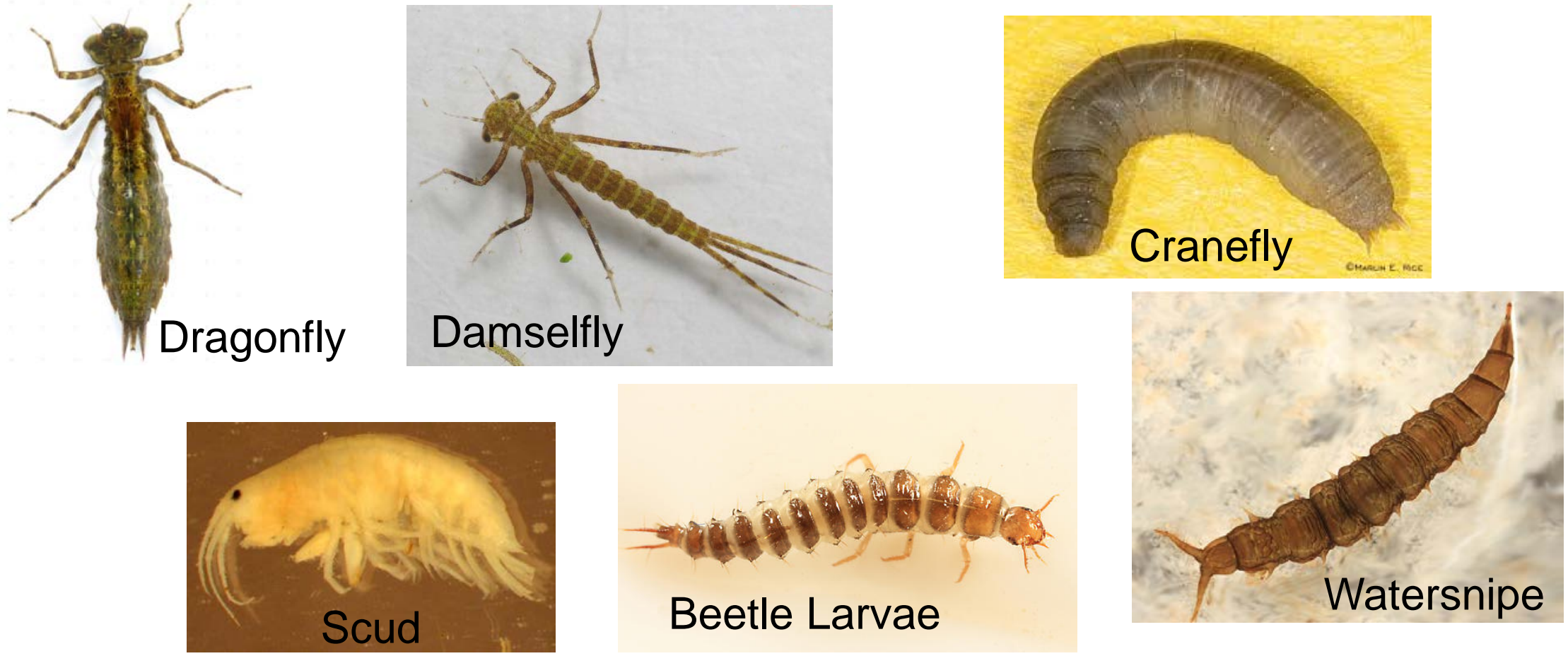


\section{Pollution Tolerant Insects}

- Aquatic worms, midges, blackflies, and more...
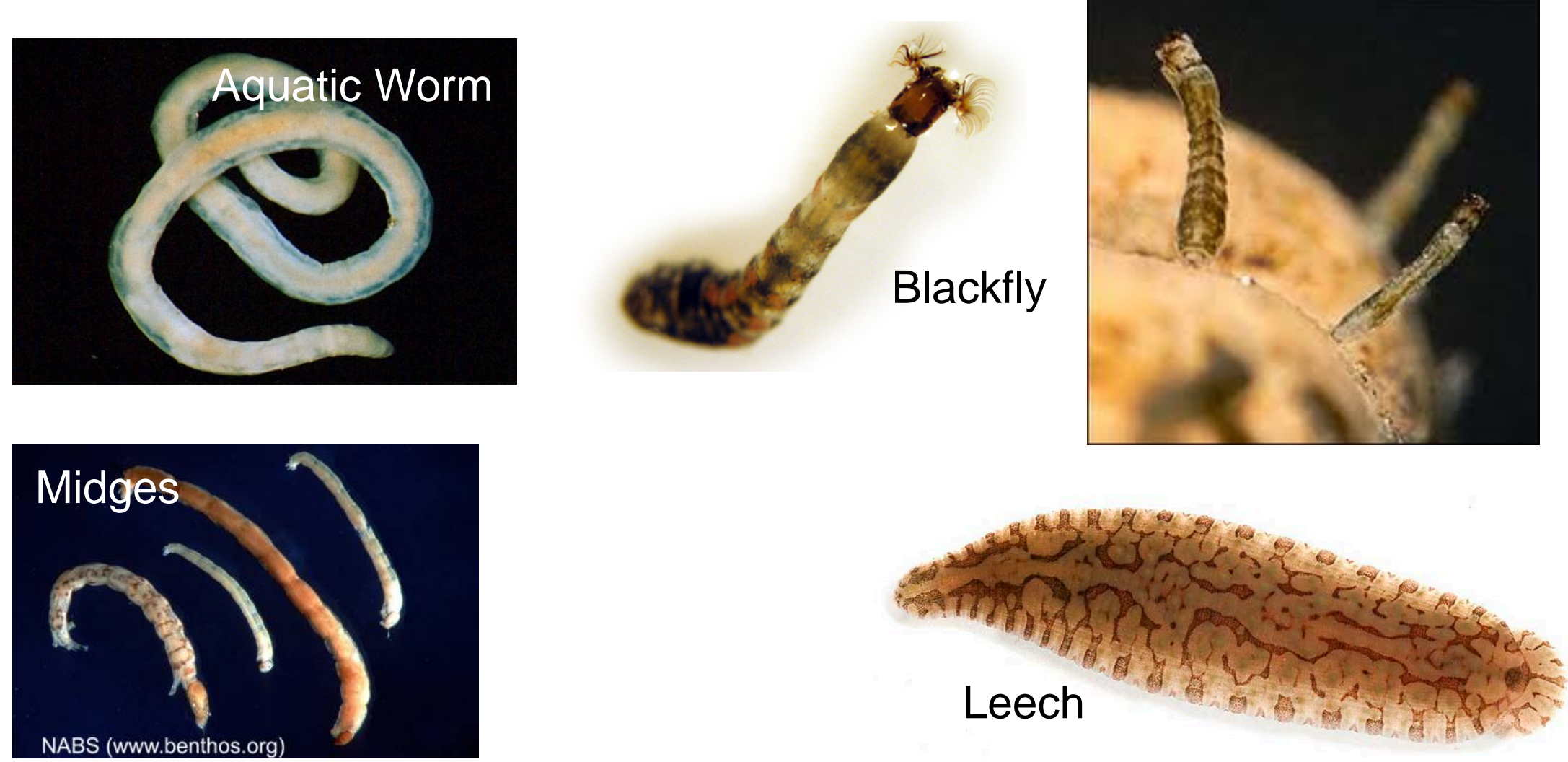

- Los Alamos

NATIONAL LABORATORY 


\section{Stream Study: Sample Record and Assessment}

\section{Macroinvertebrate Count}

\begin{tabular}{|c|c|c|}
\hline $\begin{array}{l}\text { Sensitive } \\
\square \text { caddisfly larvae } \\
\square \text { hellgrammite } \\
\square \text { mayfly larvae } \\
\square \text { gilled snail } \\
\square \text { riffle beetle adult } \\
\square \text { stonefly larvae } \\
\square \text { water penny larvae }\end{array}$ & $\begin{array}{l}\text { Somewhat Sensitive } \\
\square \text { beetle larvae } \\
\square \text { clams } \\
\square \text { cranefly larvae } \\
\square \text { crayfish } \\
\square \text { damselfly larvae } \\
\square \text { dragonfly larvae } \\
\square \text { scuds } \\
\square \text { sowbugs } \\
\square \text { fishfly larvae } \\
\square \text { alderfly larvae } \\
\square \text { watersnipe larvae }\end{array}$ & $\begin{array}{l}\text { Tolerant } \\
\square \text { aquatic worms } \\
\square \text { blackfly larvae } \\
\square \text { leeches } \\
\square \text { midge larvae }\end{array}$ \\
\hline $\begin{array}{c}\text { Total \# of boxes checked } \times 3= \\
\text { index value }\end{array}$ & $\begin{array}{r}\text { Total \# of boxes checked } 2= \\
\text { index value }\end{array}$ & $\begin{array}{c}\text { Total \# of boxes checked } 1= \\
\text { index value }\end{array}$ \\
\hline WATER QUALITY RATINC & \multicolumn{2}{|c|}{ Excellent $(>22)$} \\
\hline Total Index Value $=$ & \multicolumn{2}{|c|}{ Good (17-22) } \\
\hline
\end{tabular}




\section{Questions?}

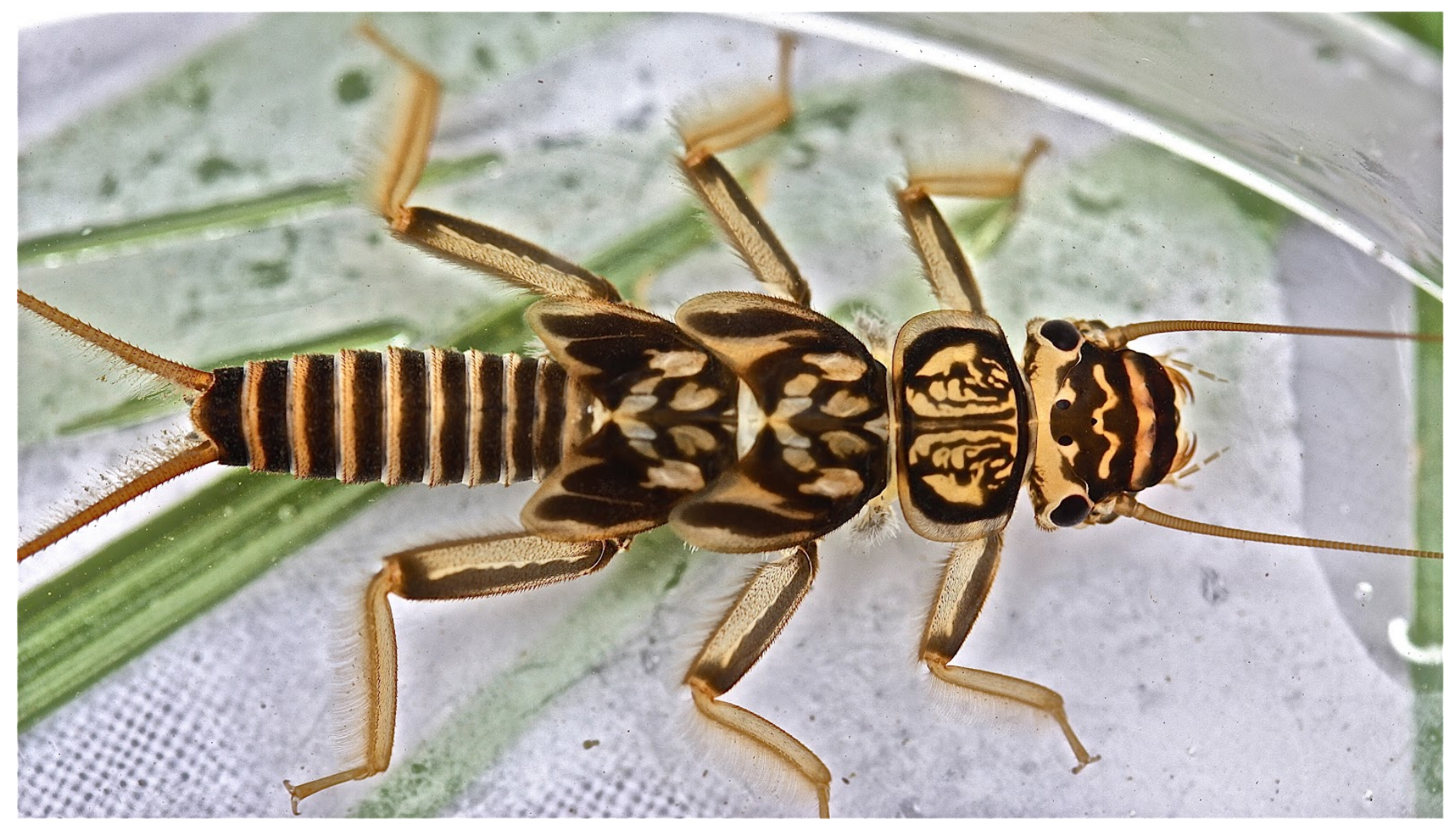

- Los Alamos NATIONAL LABORATORY 